\title{
REAL BANACH JORDAN TRIPLES
}

\author{
TRUONG C. DANG AND BERNARD RUSSO
}

(Communicated by Palle E. T. Jorgensen)

\begin{abstract}
A theory of real Jordan triples and real bounded symmetric domains in finite dimensions was developed by Loos. Upmeier has proposed a definition of a real $J B^{*}$-triple in arbitrary dimensions. These spaces include real $C^{*}$ algebras and $J B^{*}$-triples considered as vector spaces over the reals and have the property that their open unit balls are real bounded symmetric domains. This, together with the observation that many of the more recent techniques in Jordan theory rely on functional analysis and algebra rather than holomorphy, suggests that it may be possible to develop a real theory and to explore its relationship with the complex theory.

In this paper we employ a Banach algebraic approach to real Banach Jordan triples. Because of our recent observation on commutative $J B^{*}$-triples (see $\S 2$ ), we can now propose a new definition of a real $J B^{*}$-triple, which we call a $J^{*} B$-triple. Our $J^{*} B$-triples include real $C^{*}$-algebras and complex $J B^{*}$ triples. Our main theorem is a structure theorem of Gelfand-Naimark type for commutative $J^{*} B$-triples.
\end{abstract}

\section{Real Banach JoRdan tRIPLES}

Definition 1.1. A Banach Jordan triple is a real or complex Banach space $U$ equipped with a continuous bilinear (sesquilinear in the complex case) map

$$
U \times U \ni(x, y) \mapsto x \square y \in \mathscr{L}(U)
$$

such that with $\{x y z\}:=x \square y(z)$ we have

$$
\begin{aligned}
& \{x y z\}=\{z y x\}, \\
& \text { (2) }\{x, y,\{u v z\}\}+\{u,\{y x v\}, z\}=\{\{x y u\}, v, z\}+\{u, v,\{x y z\}\} \text {. }
\end{aligned}
$$

A Banach Jordan triple $U$ over $\mathbf{C}$ is said to be a $J B^{*}$-triple if

(a) for any $x \in U$ the operator $x \square x$ from $U$ to $U$ (that is, $x \square x(y)=$ $\{x x y\}, y \in U$ ) is hermitian (i.e., $\exp i t x \square x$ is an isometry for all real $t$ ) with nonnegative spectrum,

(b) the following norm condition holds

$$
\|x \square x\|=\|x\|^{2} .
$$

Received by the editors December 1, 1992; the contents of this paper were presented by the first author at the Great Plains Operator Theory Symposium, Texas A\&M University, April 1991.

1991 Mathematics Subject Classification. Primary 46J99, 17 C65. 
We note that equations (1) and (2) are the defining algebraic identities for a Jordan triple system.

Our first result was originally stated with some extra hypothesis. The authors wish to thank Jonathan Arazy for pointing this out and for suggesting the following proof.

Theorem 1.2. Let $U$ be a complex Banach Jordan triple. Suppose that

(1) $\|\{x x x\}\|=\|x\|^{3}$;

(2) $\|\{x y z\}\| \leq\|x\|\|y\|\|z\|$;

(3) $U$ is positive, i.e., $\sigma_{\mathscr{L}(U)}(x \square x) \subset[0, \infty)$ for each $x \in U$.

Then $U$ is a $J B^{*}$-triple.

Proof. We only need to show that $x \square x$ is hermitian for each $x \in U$.

Since $\delta:=i x \square x$ is a continuous derivation, $\alpha:=e^{t \delta}$ is a continuous automorphism for each real $t$. Thus for each $x \in U$

$$
\|\alpha(x)\|^{3}=\|\{\alpha(x), \alpha(x), \alpha(x)\}\|=\|\alpha(\{x x x\})\| \leq\|\alpha\|\|x\|^{3},
$$

and therefore, by iteration,

$$
\|\alpha(x)\| \leq\|\alpha\|^{1 / 3^{n}}\|x\| ;
$$

that is, $\|\alpha\| \leq 1$.

The terminology in the next definition was motivated by [1], and the spectral conditions were inspired by [7].

Definition 1.3. A $J^{*} B$-triple is a real Banach space $A$ equipped with a structure of a real Jordan triple system which satisfies

(1) $\|\{x x x\}\|=\|x\|^{3}$;

(2) $\|\{x y z\}\| \leq\|x\|\|y\|\|z\|$;

(3) $\sigma_{\mathscr{L}(A)}^{c}(x \square x) \subset[0, \infty)$ for $x \in A$;

(4) $\sigma_{\mathscr{L}(A)}^{c}(x \square y-y \square x) \subset i \mathbf{R}$ for $x, y \in A$.

The following proposition shows that over the complex field $J B^{*}$-triples are the same as $J^{*} B$-triples.

Proposition 1.4. Let $U$ be a complex Banach Jordan triple.

(a) If $U$ is a $J B^{*}$-triple, then $U$ is a $J^{*} B$-triple.

(b) Suppose that $U$, considered a real Banach Jordan triple, is a $J^{*} B$-triple. Then $U$ is a $J^{*}$-triple.

Proof. (a) A $J B^{*}$-triple satisfies (2) by [3, Corollary 3]. This, together with $\|x \square x\|=\|x\|^{2}$, implies (1). Thus it suffices to prove the two spectral conditions. Let $T$ denote either $x \square x$ or $x \square y-y \square x$. Since $U$ is a $J B^{*}$-triple, the spectrum of $T$ with respect to $\mathscr{L}(U)$ consists entirely of boundary points. Moreover, denoting by $\mathscr{L}_{\mathbf{R}}(U)$ the Banach algebra of real linear bounded operators on $U$, we trivially have $\mathscr{L}(U) \subset \mathscr{L}_{\mathbf{R}}(U)$, so $(\mathscr{L}(U))^{\mathbf{C}} \subset\left(\mathscr{L}_{\mathbf{R}}(U)\right)^{\mathbf{C}}$, and therefore, by $[4,9 D]$

$$
\sigma_{\mathscr{L}_{\mathbb{R}(U)}^{c}}^{c}(T) \subset \sigma_{(\mathscr{L}(U))} \mathbf{c}(T)=\sigma_{\mathscr{L}(U)}^{c}(T)=\sigma_{\mathscr{L}(U)}(T) \cup \overline{\sigma_{\mathscr{L}(U)}(T)} .
$$


(b) In order to apply Theorem 1.2 we only need to show that $\sigma_{\mathscr{L}(U)}(u \square u) \geq 0$ for each $u \in U$. With $T=u \square u$, we have ${ }^{1}$

$$
\sigma_{\mathscr{L}(U)}(T) \subset \sigma_{\mathscr{L}(U)}(T) \cup \overline{\sigma_{\mathscr{L}(U)}(T)}=\sigma_{(\mathscr{L}(U))} \mathrm{c}(T)=\sigma_{\left(\mathscr{L}_{\mathrm{R}}(U)\right) \mathrm{c}}(T) \subset[0, \infty) .
$$

This completes the proof.

Recall that a closed subtriple of a $J B^{*}$-triple is a $J B^{*}$-triple. Using the following fact we can give another natural example of a $J^{*} B$-triple.

Remark 1.5. A closed subtriple $B$ of a $J^{*} B$-triple $A$ is a $J^{*} B$-triple. In particular, a closed real subtriple of a $J B^{*}$-triple is a $J^{*} B$-triple.

Proof. The norm conditions are automatically satisfied, so it suffices to prove the spectral conditions.

As above, let $T$ denote either $x \square x$ or $x \square y-y \square x$ for some $x, y \in B$. We know that

$$
\sigma_{\mathscr{L}(A)}^{c}(x \square x) \subset[0, \infty) \text { and } \sigma_{\mathscr{L}(A)}^{c}(x \square y-y \square x) \subset i \mathbf{R},
$$

and we must prove

$$
\sigma_{\mathscr{L}(B)}^{c}(x \square x) \subset[0, \infty) \quad \text { and } \quad \sigma_{\mathscr{L}(B)}^{c}(x \square y-y \square x) \subset i \mathbf{R} .
$$

Let $S$ be the restriction of $T$ to $B$. Now the complexification of the restriction map is a unital complex algebra homomorphism from the algebra $\langle T, I\rangle$ generated by $T$ and $I$ into the algebra $\langle S, I\rangle$ generated by $S$ and $I$. Therefore,

$$
\sigma_{\mathscr{L}(B)}^{c}(S) \subset \sigma_{\langle S, I\rangle}^{c}(S) \subset \sigma_{\langle T, I\rangle}(T)=\sigma_{\mathscr{L}(A)}^{c}(T) .
$$

This completes the proof.

A real $C^{*}$-algebra is a closed subalgebra of its complexification, which is a complex $C^{*}$-algebra in some norm. Thus, a real $C^{*}$-algebra with the triple product

$$
\{x y z\}=\frac{1}{2}\left(x y^{*} z+z y^{*} x\right)
$$

is a closed real subtriple of a $J B^{*}$-triple. By the preceding remark, a real $C^{*}$ algebra is a $J^{*} B$-triple.

Two important problems left open in this paper are

Problem 1. Is the complexification of a $J^{*} B$-triple a $J B^{*}$-triple in some norm extending the original norm? (This is solved for commutative $J^{*} B$-triples in Theorem 3.11.)

Problem 2. Is the bidual of a $J^{*} B$-triple a $J^{*} B$-triple with a separately weak*continuous triple product?

\section{COMMUTATIVE COMPLEX TRIPLES}

In this short section we are going to use Theorem 1.2 to modify the treatment in $[5, \S 1]$ by not requiring that $x \square x$ be hermitian. Theorem 2.2 will be used to prove the main result of this paper, namely Theorem 3.11 , which leads to a Gelfand-Naimark Theorem for commutative real $J^{*} B$-triples.

\footnotetext{
${ }^{1}$ Note that the second spectral condition is not used.
} 
Definition 2.1. A Banach Jordan triple is commutative if

$$
\{\{x y z\} u v\}=\{x y\{z u v\}\}=\{x\{y z u\} v\} .
$$

For example, any commutative $C^{*}$-algebra $C_{0}(\Omega)$ is a commutative Banach Jordan triple with $f \square g(h)=f \bar{g} h$.

From (1) and (4) we have

$$
\{x y z\} \square u=x \square\{y z u\}=(x \square y)(z \square u) .
$$

By the continuity assumption, there is a constant $N$ such that

$$
\|\{x y z\}\| \leq N\|x\|\|y\|\|z\| .
$$

Throughout this section $U$ will denote a commutative complex Banach Jordan triple.

Let $B=B(U):=$ the closed span of $U \square U$ in $\mathscr{L}(U)$. Then $B$ is a commutative Banach subalgebra of $\mathscr{L}(U)$. Denote the Gelfand Transform of $B$ by

$$
B(U) \stackrel{\Gamma_{B}}{\longrightarrow} C_{0}(X)
$$

where $X=X_{B}$ is the maximal ideal space of $B$. Let $\Lambda=\Lambda(U):=$ the set of all nonzero triple homomorphisms $\lambda: U \rightarrow \mathbf{C}$. Precisely,

$$
\Lambda=\{\lambda: U \rightarrow \mathbf{C}: 0 \neq \lambda \text { linear }, \lambda(\{a b c\})=\lambda(a) \overline{\lambda(b)} \lambda(c)\} .
$$

According to [5, Lemma 1.6], $\Lambda \subset \mathscr{L}(U, \mathbf{C})$ and $\|\lambda\| \leq \sqrt{N}$, where $N$ is defined in (6). Thus $\Lambda$ is a weak*-locally compact space and a "principle T-bundle" ( $\mathbf{T}=$ unit circle) under the action

$$
\mathbf{T} \times \Lambda \ni(t, \lambda) \mapsto t \cdot \lambda \in \Lambda,
$$

where $(t \cdot \lambda)(x)=t \lambda(x)$. Moreover, there is a bijection

$$
\Lambda / \mathbf{T} \leftrightarrow\left\{I^{\text {ideal }} \subset U: U / I \cong \mathbf{C} \text { as triples }\right\}
$$

Define a norm closed subtriple of $C_{0}(\Lambda)$

$$
C_{\text {hom }}(\Lambda):=\left\{f \in C_{0}(\Lambda): f(t \cdot \lambda)=t f(\lambda) \forall(t, \lambda) \in \mathbf{T} \times \Lambda\right\}
$$

and a Gelfand transform $U \ni x \mapsto \hat{x}=\Gamma_{U}(x) \in C_{\text {hom }}(\Lambda)$ by $\Gamma_{U}(x)(\lambda)=\lambda(x)$. Thus

$$
U \stackrel{\Gamma_{U}}{\longrightarrow} C_{\mathrm{hom}}(\Lambda)
$$

is a continuous triple homomorphism.

According to [5, Lemma 1.8], $\widehat{U}$ is dense in $C_{\text {hom }}(\Lambda)$.

Theorem 2.2. Let $U$ be a commutative complex Banach Jordan triple. Suppose that

(1) $\|\{x x x\}\|=\|x\|^{3}$;

(2) $\|\{x y z\}\| \leq\|x\|\|y\|\|z\|$;

(3) $U$ is positive, i.e., $\sigma_{\mathscr{L}(U)}(x \square x) \subset[0, \infty)$ for each $x \in U$. 
Then the Gelfand representation $U \rightarrow C_{\mathrm{hom}}(\Lambda)$ is an isometric surjective triple isomorphism.

Proof. By Theorem 1.2, $U$ is a $J B^{*}$-triple, so the result follows from [5].

For a generalization of this theorem see [2].

\section{Commutative ReAl TRIPLES}

In this section $A$ will be a commutative real Banach Jordan triple, that is, a real Banach space $A$, together with a trilinear map

$$
A \times A \times A \ni(x, y, z) \mapsto\{x y z\} \in A
$$

which satisfies

$$
\begin{gathered}
\{x y z\}=\{z y x\}, \\
\{\{x y z\} u v\}=\{x y\{z u v\}\}=\{x\{y z u\} v\} .
\end{gathered}
$$

We shall define a natural Gelfand transform and prove a representation theorem of Gelfand-Naimark type.

By analogy with the complex case, let $B(A)$ be the Banach subalgebra of $\mathscr{L}(A)$ generated by $A \square A$. Then $B(A)$ is a commutative real Banach algebra (not necessarily unital, cf. [4, p. 63]). Let $X_{B(A)}^{c}$ denote the space of complexified characters (cf. [4, p. 82]); that is,

$$
X_{B(A)}^{c}=\{\tau: B(A) \rightarrow \mathrm{C}, 0 \neq \tau \text { real-linear, } \tau(S T)=\tau(S) \tau(T)\} .
$$

By analogy we define $\Lambda_{A}^{c}$ to be the collection of all nonzero real-linear triple homomorphisms of $A$ into $\mathbf{C}$; precisely,

$$
\Lambda_{A}^{c}=\{\lambda: A \rightarrow \mathbf{C}: \lambda \text { real linear, } \lambda \neq 0, \lambda(\{a b c\})=\lambda(a) \overline{\lambda(b)} \lambda(c)\} .
$$

By the proof of [5, Lemma 1.6], each such $\lambda$ is automatically continuous and $\Lambda_{A}^{c}$ is contained in a bounded subset of $\mathscr{L}_{\mathbf{R}}(A, \mathbf{C})$. Note that $e^{i \theta} \Lambda_{A}^{c}=\Lambda_{A}^{c}$, that $\Lambda_{A}^{c}$ is closed under complex conjugation, and that $\Lambda_{A}^{c}$ is locally compact in the topology of pointwise convergence on $A$.

Of course, we shall occasionally make use of the box operators $x \square y: a \mapsto$ $\{x y a\}$.

In order to obtain the analogue of Theorem 2.2 we need to consider the complexification of $A$.

Let $U:=A^{\mathrm{C}}=\phi(A)+i \phi(A)$ be the complexification of $A$, and let $\phi: A \rightarrow U$ be the natural embedding. The space $U$ becomes a complex commutative Jordan triple system in the natural way, and $\phi$ is a real-linear triple isomorphism into. Explicitly, $U=A \times A$ becomes a complex linear space under $(\alpha+i \beta)(x, y)=(\alpha x-\beta y, \alpha y+\beta x), \alpha, \beta \in \mathbf{R}, x, y \in A$, and $\phi(a)=(a, 0)$ for $a \in A$. Also, if $x, y, z \in U$ and we write $x=x_{1}+i x_{2}$ for $\left(x_{1}, x_{2}\right)$ and so on, then

$$
\begin{aligned}
\{x y z\}_{U}= & \left\{x_{1} y_{1} z_{1}\right\}_{A}+\left\{x_{1} y_{2} z_{2}\right\}_{A}+\left\{x_{2} y_{2} z_{1}\right\}_{A}-\left\{x_{2} y_{1} z_{2}\right\}_{A} \\
& +i\left(\left\{x_{2} y_{1} z_{1}\right\}_{A}-\left\{x_{1} y_{2} z_{1}\right\}_{A}+\left\{x_{1} y_{1} z_{2}\right\}_{A}+\left\{x_{2} y_{2} z_{2}\right\}_{A}\right) .
\end{aligned}
$$

We will use the given norm on $A$ to define a norm on $U$ so that we can impose further assumptions on the spectrum of certain operators. We give $U$ 
a preliminary norm as defined in $[4,(9.1)]$

$$
\|u\|_{2}:=\frac{1}{\sqrt{2}} \sup \left\{\|\lambda u\|_{1}: \lambda \in \mathbf{T}\right\},
$$

where if $u=(x, y)=\phi(x)+i \phi(y) \in U$,

$$
\|u\|_{1}=\|x\|+\|y\| \text {. }
$$

Recall that $\left(U,\|\cdot\|_{1}\right)$ is a real Banach space, $\left(U,\|\cdot\|_{2}\right)$ is a complex Banach space, $\phi$ is an isometry in each norm, and $(1 / \sqrt{2})\|\cdot\|_{1} \leq\|\cdot\|_{2} \leq\|\cdot\|_{1}$. For $T \in \mathscr{L}(U)=\mathscr{L}\left(U,\|\cdot\|_{2}\right)$ let $\|T\|_{\mathscr{L}(U)}$ denote the operator norm

$$
\|T\|_{\mathscr{L}(U)}=\sup _{u \neq 0, u \in U}\left(\|T u\|_{2} /\|u\|_{2}\right) \text {. }
$$

Lemma 3.1. $U \square U \subset \mathscr{L}(U)$ and $\|x \square y\|_{\mathscr{L}(U)} \leq 2^{3 / 2}\|x\|_{2}\|y\|_{2}$ for $x, y \in U$. Thus $U$ is a commutative complex Banach Jordan triple.

Proof. If $a, b \in A$ and $z=z_{1}+i z_{2} \in U$, then

$$
\begin{aligned}
\|(a \square b) z\|_{2} & \leq\|(a \square b) z\|_{1}=\left\|\left\{a b z_{1}\right\}\right\|_{A}+\left\|\left\{a b z_{2}\right\}\right\|_{A} \leq\|a\|_{A}\|b\|_{A}\|z\|_{1} \\
& \leq\|a\|_{A}\|b\|_{A} \sqrt{2}\|z\|_{2},
\end{aligned}
$$

so $\|a \square b\|_{\mathscr{L}(U)} \leq \sqrt{2}\|a\|_{A}\|b\|_{A}$.

In general, $\left(x_{1}+i x_{2}\right) \square\left(y_{1}+i y_{2}\right)=x_{1} \square y_{1}-x_{2} \square y_{2}+i\left(x_{2} \square y_{1}\right)-i\left(x_{1} \square y_{2}\right)$ now implies that $\left\|\left(x_{1}+i x_{2}\right) \square\left(y_{1}+i y_{2}\right)\right\| \leq \sqrt{2}\left\|x_{1}+i x_{2}\right\|_{1}\left\|y_{1}+i y_{2}\right\|_{1} \leq$ $2^{3 / 2}\left\|x_{1}+i x_{2}\right\|_{2}\left\|y_{1}+i y_{2}\right\|_{2}$.

As in $\S 2$, let $B(U)$ be the closed complex subalgebra of $\mathscr{L}(U)$ generated by $U \square U$ and define $B(\phi(A))$ to be closed real subalgebra of $\mathscr{L}(U)$ generated by $\phi(A) \square \phi(A)$.

Lemma 3.2. $B(\phi(A))$ is a real Banach algebra which is isometrically isomorphic to $B(A)$.

Proof. The map $\sigma: \operatorname{sp}_{\mathbf{R}} A \square A \rightarrow \operatorname{sp}_{\mathbf{R}} \phi(A) \square \phi(A)$ defined by $\sum x_{i} \square y_{i} \mapsto$ $\sum \phi\left(x_{i}\right) \square \phi\left(y_{i}\right)$ is an isomorphism which is isometric, so it extends to the desired isomorphism. To see this, let $X=\sum x_{i} \square y_{i}$ and $u=\phi(a)+i \phi(b) \in U$. Then $\sigma(X) u=\phi(X a)+i \phi(X b)$, so $\|\sigma(X) u\|_{1} \leq\|X\|_{\mathscr{L}(A)}\|u\|_{1}$. Since $\sigma(X)$ is complex linear,

$$
\begin{aligned}
\|\sigma(X) u\|_{2} & =\frac{1}{\sqrt{2}} \sup _{\lambda}\|\lambda \sigma(X) u\|_{1}=\frac{1}{\sqrt{2}} \sup _{\lambda}\|\sigma(X)(\lambda u)\|_{1} \\
& \leq \frac{1}{\sqrt{2}} \sup _{\lambda}\|x\|\|\lambda u\|_{1}=\|X\|\|u\|_{2} .
\end{aligned}
$$

Hence $\|\sigma(X)\|_{\mathscr{L}(U)} \leq\|X\|_{\mathscr{L}(A)}$.

Pick $a \in A$ of norm 1 such that the norm of $X a$ is close to the norm of $X$. Then $u=\phi(a)$ satisfies $\|u\|_{2}=1$ and $\|\sigma(X) u\|_{2}=\|X a\|_{A}$, showing that $\sigma$ is isometric and hence well defined. It is trivial that $\sigma$ is a homomorphism.

Lemma 3.3. For $T, S \in B(\phi(A))$ we have

$$
\|T\|_{\mathscr{L}(U)} \leq \sqrt{2}\|T+i S\|_{\mathscr{L}(U)} .
$$


Proof. For $x \in A$ both $T(\phi(x))$ and $S(\phi(x))$ belong to $\phi(A)$, so

$$
\|(T+i S)(\phi(x))\|_{1}=\|T(\phi(x))+i S(\phi(x))\|_{1}=\|T(\phi(x))\|_{2}+\|S(\phi(x))\|_{2} .
$$

Thus $\|T(\phi(x))\|_{2} \leq\|(T+i S)(\phi(x))\|_{1} \leq \sqrt{2}\|(T+i S) \phi(x)\|_{2}$. Hence

$$
\|T\|_{\mathscr{L}(U)}=\left\|\sigma^{-1}(T)\right\|_{\mathscr{L}(A)}=\left\|\left.T\right|_{\phi(A)}\right\|_{\mathscr{L}(\phi(A), U)} \leq \sqrt{2}\|T+i S\|_{\mathscr{L}(U)} .
$$

Proposition 3.4. Let $A$ be a commutative real Banach Jordan triple. The complex subalgebras $B(U)$ and $B(\phi(A))+i B(\phi(A))$ of $\mathscr{L}(U)$ coincide. Thus $B(U)$ is the complexification $B(\phi(A))^{\mathbf{C}}$ of $B(\phi(A))$. Less precisely, $B\left(A^{\mathbf{C}}\right)=(B(A))^{\mathbf{C}}$. Proof. It is clear that $\mathrm{sp}_{\mathrm{C}} U \square U \subset B(\phi(A))+i B(\phi(A)) \subset B(U)$. To complete the proof is suffices to show that $B(\phi(A))+i B(\phi(A))$ is a closed subset of $\mathscr{L}(U)$, but this follows immediately from Lemma 3.3.

The following lemma is based on some ideas from [8].

Lemma 3.5. Let $A$ be a commutative real Banach Jordan triple.

(a) Suppose that $\{x x x\}=0$ implies $x=0$. Then $\left(\sum_{1}^{n} x_{i} \square y_{i}\right)^{*}:=\sum_{1}^{n} y_{i} \square x_{i}$ defines an involution on the linear span of $A \square A$ (i.e., a linear automorphism of order 2).

(b) Suppose further that $\|\{x x x\}\|=\|x\|^{3}$ and

$$
\|\{x y z\}\| \leq\|x\|\|y\|\|z\| \text {. }
$$

Then the map * defined in (a) extends to an involution on $B(A)$ which makes $B(A)$ into a real Banach *-algebra satisfying all the axioms of a real $C^{*}$-algebra except possibly for the invertibility of $I+T^{*} T$ in the unital extension.

Proof. (a) We first show, as in [8], that ${ }^{*}$ is well defined. For this, suppose that $\sum_{1}^{n} x_{i} \square y_{i}=0$ and let $T=\sum_{1}^{n} y_{i} \square x_{i}$. To show that $T=0$, let $z \in A$ and $u_{j}=\left\{y_{j} x_{j} z\right\}$. Then $T z=\sum\left\{y_{i} x_{i} z\right\}$, and

$$
\begin{aligned}
\{T z, T z, T z\} & =\sum_{i, j, k}\left\{\left\{y_{i} x_{i} z\right\},\left\{y_{j} x_{j} z\right\},\left\{y_{k} x_{k} z\right\}\right\} \\
& =\sum_{i, j, k}\left\{\left\{z x_{i} y_{i}\right\} u_{j} u_{k}\right\}=\sum_{i, j, k}\left\{z\left\{x_{i} y_{i} u_{j}\right\} u_{k}\right\} \\
& =\sum_{j, k}\left\{z, \sum_{i}\left\{x_{i} y_{i} u_{j}\right\}, u_{k}\right\}=\sum_{j, k}\left\{z, 0, u_{k}\right\}=0 .
\end{aligned}
$$

Then by assumption $T z=0$, so $T=0$ and ${ }^{*}$ is well defined. Clearly, * is linear and of order 2. To prove that $(T S)^{*}=T^{*} S^{*}$, it suffices by additivity to assume that $T, S \in A \square A$. With $T=x \square y$ and $S=z \square w$ we have $(T S)^{*}=((x \square y)(z \square w))^{*}=(x \square\{y z w\})^{*}=\{y z w\} \square x=\{w z y\} \square x$ and $S^{*} T^{*}=(w \square z)(y \square x)=\{w z y\} \square x$, by $(5)$.

(b) We again argue as in [8]. Let $S=\sum_{1}^{n} x_{i} \square y_{i}$ and $z \in A$. Then

$$
\begin{aligned}
\|S z\|^{3} & =\|\{S z, S z, S z\}\| \\
& =\left\|\sum_{i}\left\{S z, S z,\left\{x_{i} y_{i} z\right\}\right\}\right\|=\left\|\sum_{i}\left\{S z,\left\{S z, x_{i}, y_{i}\right\}, z\right\}\right\| \\
& =\left\|\left\{S z, S^{*} S z, z\right\}\right\| \leq\|S z\|\left\|S^{*} S z\right\|\|z\| .
\end{aligned}
$$


Therefore, $\|S\|^{2} \leq\left\|S^{*} S\right\|$, which implies that ${ }^{*}$ is isometric and extends to a $C^{*}$-involution on $B$.

In order to proceed further, we shall need to make some spectral assumptions.

Lemma 3.6. Let $A$ be a commutative real Banach Jordan triple. Suppose that $\{x x x\}=0$ implies $x=0$, and assume the two spectral conditions

$$
\begin{array}{cl}
\sigma_{\mathscr{L}(A)}^{c}(x \square x) \subset[0, \infty) & \text { for } x \in A, \\
\sigma_{\mathscr{L}(A)}^{c}(x \square y-y \square x) \subset i \mathbf{R} & \text { for } x, y, \in A .
\end{array}
$$

For each $\tau \in X_{B(A)}^{c}$ there is $\lambda \in \Lambda_{A}^{c}$ such that $\tau(x \square y)=\lambda(x) \overline{\lambda(y)}$ for $x, y \in$ $A$. This correspondence establishes a bijection

$$
X_{B(A)}^{c} \leftrightarrow \Lambda_{A}^{c} / \mathbf{T} \text {. }
$$

Proof. If $\lambda \in \Lambda_{A}^{c}$, there exist $t \in \mathbf{R}$ and $\alpha \in A$ such that $e^{i t} \lambda(\alpha)=1$. Define $\tau: B(A) \rightarrow \mathbf{C}$ by $\tau(T)=e^{i t} \lambda(T \alpha)$. Then

$$
\tau(x \square y)=\left(e^{i t} \lambda\right)(\{x y \alpha\})=\lambda(x) \overline{\lambda(y)} .
$$

Note that $\tau$ does not depend on the choices of $t$ and $\alpha$.

We next prove that $\tau(T S)=\tau(T) \tau(S)$ for all $T, S \in B(A)$. By continuity and linearity, it suffices to prove this for $T, S \in A \square A$. With $T=x \square y$ and $S=z \square w$ we have $T S=x \square\{y z w\}$ and

$$
\tau(T S)=\lambda(x) \overline{\lambda(\{y z w\})}=\lambda(x) \overline{\lambda(y)} \lambda(z) \overline{\lambda(w)}=\tau(T) \tau(S) .
$$

Thus $\tau \in X_{B(A)}^{c}$, and it is clear that $e^{i \theta} \lambda$ and $\lambda$ give rise to the same $\tau$. This gives a map $\lambda \mapsto \tau$ from $\Lambda_{A}^{c} / T$ to $X_{B(A)}^{c}$.

Now let $\tau \in X_{B(A)}^{c}$. By the nonunital version of $[4,10.4]$,

$$
\tau(x \square x) \in \sigma_{B(A)}^{c}(x \square x)=\sigma_{B(A) \mathrm{c}}(x \square x) \supset \sigma_{\mathscr{L}(A) \mathrm{c}}(x \square x)=\sigma_{\mathscr{L}(A)}^{c}(x \square x) .
$$

However, by (10), equality holds, and therefore, for all $x \in A, \tau(x \square x) \geq 0$.

We claim that there exists an $\alpha \in A$ such that $\tau(\alpha \square \alpha)=1$, for otherwise we would have $\tau(x \square x)=0$ for all $x \in A$ and thus $\tau((x+y) \square(x+y))=0$, implying

$$
\tau(x \square y+y \square x)=0 \quad \text { for all } x, y \in A .
$$

From Lemma 3.5 and (12) it follows that $\tau\left(T^{*}\right)=-\tau(T)$ for all $T \in B(A)$ and hence that $\tau\left(T^{2}\right)=(\tau(T))^{2}=\left(\tau\left(T^{*}\right)\right)^{2}=\tau\left(\left(T^{2}\right)^{*}\right)=-\tau\left(T^{2}\right)$. Thus $\tau(T)=0$ identically on $B(A)$, a contradiction. Thus such an $\alpha$ exists.

Now define a linear functional $\lambda$ by $\lambda(x)=\tau(x \square \alpha)$ for $x \in A$. We shall show that $\lambda \in \Lambda_{A}^{c}$ and $\lambda(T \alpha)=\tau(T)$ for all $T \in B(A)$, thereby demonstrating the surjectivity of the map $\lambda \mapsto \tau$.

The second statement follows from (5); with $T=x \square y$, we have

$$
\lambda(T \alpha)=\tau(T \alpha \square \alpha)=\tau(x \square y \alpha \square \alpha=\tau(T)) .
$$

To prove the first statement we first prove

$$
\tau(x \square y)=\overline{\tau(y \square x)} \text { for } x, y \in A,
$$


for then, if $a, b, c, \in A$,

$$
\begin{aligned}
\lambda\{a b c\} & =\tau(\{a b c\} \square \alpha)=\tau((a \square b)(c \square \alpha)) \\
& =\tau((a \square b)(\alpha \square \alpha(c \square \alpha))=\tau((a \square \alpha)(\alpha \square b)(c \square \alpha)) \\
& =\tau(a \square \alpha) \tau\left((b \square \alpha)^{*}\right) \tau(c \square \alpha)=\lambda(a) \overline{\lambda(b)} \lambda(c),
\end{aligned}
$$

i.e., $\lambda \in \Lambda$.

It remains to prove (13). Since $x \square y+y \square x=(x+y) \square(x+y)-x \square x-y \square y$, we have $\tau(x \square y)+\tau(y \square x)$ is real. Since $(x \square y)(y \square x)=(x \square x)(y \square y)$, we have $\tau(x \square y) \tau(y \square x) \geq 0$. From these two relations, (13) follows in case $\tau(x \square y)$ is not a real number. On the other hand, if $\tau(x \square y)$ is a real number, then so is $\tau(y \square x)$ and the difference $\tau(x \square y)-\tau(y \square x)$ is both real and purely imaginary by (11). This proves (13).

In Proposition 3.7, under additional assumptions, $B(A)$ will be shown to be a real $C^{*}$-algebra.

Proposition 3.7. Suppose that $A$ is a commutative $J^{*} B$-triple. Then $B(A)$, with the norm of $\mathscr{L}(A)$, is a commutative real $C^{*}$-algebra with involution determined by $(x \square y)^{*}=y \square x$. Consequently, $B(U)$ is a $C^{*}$-algebra in some norm extending the norm on $B(A)$ (by $[4,12.4])$.

Proof. By Lemma 3.5 all the properties of a real $C^{*}$-algebra are satisfied except possibly the invertibility of elements of the form $1+T^{*} T$ in the unital extension $B(A)+\mathbf{R} I d_{A}$ of $B(A)$. For $T=S+\lambda I d_{A}$ it suffices to show that $\sigma_{B(A)}^{c}\left(T^{*} T\right) \geq$ 0 , which in turn will follow from the fact that $\tau\left(T^{*} T\right) \geq 0$ for all $\tau \in X_{B(A)}^{c}$. By Lemma 3.6, $\tau\left(S^{*}\right)=\overline{\tau(S)}$ for all $S \in B(A)$, and the same holds for $T \in$ $B(A)+\mathbf{R} I d_{A}$. Thus $\tau\left(T^{*} T\right)=|\tau(T)|^{2} \geq 0$.

The following lemma will be used in the proof of Theorem 3.11 only.

Lemma 3.8. Denote the norm in the $C^{*}$-algebra $B(U)$ by $\|\cdot\|_{C^{*}}$. For any selfadjoint $G$ in $B(U)$ we have

$$
\|G\|_{\mathscr{L}(U)} \leq 4 \sqrt{2}\|G\|_{C^{*}} .
$$

Proof. Let $G=T+i S$ with $T, S \in B(A)$. By [4, 12.2(a)],

$$
\|T\| \leq\left\|T^{*} T+S^{*} S\right\|^{1 / 2}
$$

and similarly for $\|S\|$. Thus $\|G\|_{\mathscr{L}(U)} \leq\|T\|_{B(A)}+\|S\|_{B(A)} \leq 2\left\|T^{*} T+S^{*} S\right\|_{\mathscr{L}(U)}^{1 / 2}$.

On the other hand, $G^{2}=G^{*} G=\left(T^{*} T+S^{*} S\right)+i\left(T^{*} S-S^{*} T\right)$ implies (by Lemma 3.3) $\left\|T^{*} T+S^{*} S\right\|_{\mathscr{L}(U)} \leq \sqrt{2}\left\|G^{*} G\right\|_{\mathscr{L}(U)}$. Thus $\|G\|_{\mathscr{L}(U)}^{2} \leq 4 \sqrt{2}\left\|G^{*} G\right\|$ $=4 \sqrt{2}\left\|G^{2}\right\|$. With $\beta=4 \sqrt{2}$, induction shows that $\|G\|_{\mathscr{L}(U)}^{2^{n}} \leq \beta^{2^{n}-1}\left\|G^{2^{n}}\right\|$. Finally, $\|G\|_{\mathscr{L}(U)}=\left(\|G\|^{2^{n}}\right)^{1 / 2^{n}} \leq\left(\beta^{2^{n}-1}\right)^{1 / 2^{n}}\left\|G^{2^{n}}\right\|^{1 / 2^{n}}$, and therefore $\|G\| \leq$ $\beta \lim \left\|G^{2^{n}}\right\|^{1 / 2^{n}}=\beta\|G\|$.

Let $\Lambda(U)$ be defined as in $\S 2$.

Lemma 3.9. With the above notation,

(i) For each $\lambda \in \Lambda(U)$ there is $\lambda^{\prime} \in \Lambda_{A}^{c}$ such that $\lambda(\phi(x)+i \phi(y))=$ $\lambda^{\prime}(x)+i \lambda^{\prime}(y)$ for $x, y \in A$. This correspondence establishes a bijection $\Lambda(U) \leftrightarrow \Lambda_{A}^{c}$. 
(ii) For each $\tau \in X_{B(U)}$ there is $\tau^{\prime} \in X_{B(A)}^{c}$ such that $\tau(T+i S)=\tau^{\prime}(T)+$ $i \tau^{\prime}(S)$ for $T, S \in B(A)$. This correspondence establishes a bijection $X_{B(U)} \leftrightarrow X_{B(A)}^{c}$.

Proof. The second part is precisely the statement of the nonunital version of $[4,(10.6)]$, and the first part is proved in exactly the same way.

Lemma 3.10. For each $\tau \in X_{B(U)}$ there is $\lambda \in \Lambda(U)$ such that $\tau(u \square v)=$ $\lambda(u) \overline{\lambda(v)}$ for $u, v \in U$. This correspondence establishes a bijection $X_{B(U)} \leftrightarrow$ $\Lambda(U) / \mathbf{T}$.

Proof. Lemmas 3.9 and 3.6.

We can now state and prove the main result of this paper.

Theorem 3.11. Let $A$ be a commutative $J^{*} B$-triple. There is a norm on the complexification $U$ of $A$ extending the norm on $A$ and for which $U$ is a $J B^{*}$ triple.

Proof. First we note that the norm condition $\|\{x x x\}\|=\|x\|^{3}$ and (9) imply that, for $x \in A,\|x\|^{2}=\|x \square x\|$. By Proposition 3.7 and Lemma 3.6,

$$
\|x\|^{2}=\sup \left\{|\tau(x \square x)|: \tau \in X_{B(A)}^{c}\right\}=\sup \left\{|\lambda(x)|^{2} ; \lambda \in \Lambda_{A}^{c}\right\} .
$$

Define a function $\|\cdot\|_{3}$ on $U$ by $\|u\|_{3}=\sup \{|\lambda(u)|: \lambda \in \Lambda(U)\}$. Note that $\|u\|_{3}^{2}=\sup _{\tau \in X_{B(U)}} \tau(u \square u)=\|u \square u\|_{C^{*}}$. By Lemma 3.9(i), $\|\phi(a)\|_{3}=\|a\|_{A}$ if $a \in A$. It is obvious that $\|\cdot\|_{3}$ is a seminorm satisfying properties (1) and (2) of Theorem 2.2. Suppose that $\|u\|_{3}=0$. Then $\tau(u \square u)=0$ for all $\tau \in X_{B(U)}$, so $u \square u=0$. Writing $u=x+i y$ with $x, y \in \phi(A)$, we have $0=x \square x+y \square y+i(y \square x-x \square y)$, which implies $x \square x+y \square y=0$, and hence $x=y=0$ (since $B(A)$ is a real $C^{*}$-algebra). Thus we have a norm, and we next prove that (3) of Theorem 2.2 is satisfied. For this it suffices to show that $\sigma_{B(U)}(u \square u) \geq 0$. Since $B(U)$ is a $C^{*}$-algebra, $\sigma_{B(U)}(u \square u)=\{\tau(u \square u): \tau \in$ $\left.X_{B(U)}\right\}$, and by Lemma 3.10 this is $\geq 0$.

It remains to show that $U$ is complete in the norm $\|\cdot\|_{3}$. This will follow from the following: if $u=x+i y$ with $x, y \in A$, then $\|x\|_{3} \leq 2 \sqrt{2}\|u\|_{3}$. To prove this, write $u \square u=(x \square x+y \square y)+i(y \square x-x \square y)$, which implies (by Lemmas 3.3 and 3.8) $\|x \square x+y \square y\|_{\mathscr{L}(U)} \leq \sqrt{2}\|u \square u\|_{\mathscr{L}(U)} \leq 8\|u \square u\|_{C^{*}}$. Since $x \square x$ and $y \square y$ belong to the real $C^{*}$-algebra $B(A),\|x \square x\|_{B(A)} \leq$ $\|x \square x+y \square y\|_{B(A)} \leq 8\|u \square u\|_{C^{*}}$. Thus

$$
\|x\|_{3}^{2}=\|x\|_{A}^{2}=\|x \square x\|_{B(A)} \leq 8\|u \square u\|_{C^{*}}=8\|u\|_{3}^{2},
$$

completing the proof.

We conclude by describing the Gelfand transform and proving a GelfandNaimark type theorem for commutative $J^{*} B$-triples.

As noted earlier, the space $\Lambda_{A}^{c}$ is a locally compact Hausdorff space in the topology of pointwise convergence on $A$. The bijection in Lemma 3.9(i) is a homeomorphism. Now let

$$
C_{\mathrm{hom}}^{*}\left(\Lambda_{A}^{c}\right)=\left\{f \in C_{0}\left(\Lambda_{A}^{c}\right): f\left(e^{i \theta} \lambda^{\prime}\right)=e^{i \theta} f\left(\lambda^{\prime}\right) \text { and } f\left(\overline{\lambda^{\prime}}\right)=\overline{f\left(\lambda^{\prime}\right)}\right\},
$$


and define a Gelfand transform $\Gamma_{A}^{\mathbf{R}}: A \rightarrow C_{\text {hom }}^{*}\left(\Lambda_{A}^{c}\right)$ by $\Gamma_{A}^{\mathbf{R}}(x)\left(\lambda^{\prime}\right)=\lambda^{\prime}(x)$. Let $\rho: \Lambda_{U} \rightarrow \Lambda_{A}^{c}$ be the restriction map used in Lemma 3.9, and let $\rho^{*}: C_{\text {hom }}^{*}\left(\Lambda_{A}^{c}\right) \rightarrow$ $C_{\text {hom }}\left(\Lambda_{U}\right)$ be its transpose.

Note that $\rho^{-1}(\bar{\lambda})(\phi(x)+i \phi(y))=\overline{\lambda(x)}+i \overline{\lambda(y)}$, and therefore $\Gamma_{A}^{\mathbf{R}}$ maps $A$ into $C_{\text {hom }}^{*}\left(\Lambda_{A}^{c}\right)$.

Since

$$
\Gamma_{U}^{\mathbf{C}} \circ \phi=\rho^{*} \circ \Gamma_{A}^{\mathbf{R}}
$$

$\Gamma_{A}^{\mathbf{R}}$ is an isometry.

Finally, if $f \in C_{\text {hom }}^{*}\left(\Lambda_{A}^{c}\right)$ and $x, y \in A$ are such that

$$
\rho^{*} f=\Gamma_{U}(\phi(x)+i \phi(y)),
$$

the fact that $f\left(\overline{\lambda^{\prime}}\right)=\overline{f\left(\lambda^{\prime}\right)}$ implies that $y=0$; hence $\Gamma_{A}^{\mathbf{R}}(A)=C_{\text {hom }}^{*}\left(\Lambda_{A}^{c}\right)$. This proves

Theorem 3.12. Let $A$ be a commutative $J^{*} B$-triple. Then the Gelfand transform is an isometric triple isomorphism of $A$ onto $C_{\mathrm{hom}}^{*}\left(\Lambda_{A}^{c}\right)$.

\section{REFERENCES}

1. K. Alvermann, Real normed Jordan Banach algebras with an involution, Arch. Math. (Basel) 47 (1986), 135-150.

2. Y. Friedman and B. Russo, Function representation of commutative operator triple systems, J. London Math. Soc. (2) 27 (1983), 513-524.

3. _ _ The Gelfand Naimark theorem for JB*-triples, Duke Math. J. 53 (1986), 139-148.

4. K. R. Goodearl, Notes on real and complex $C^{*}$-algebras, Shiva Publishing, England, 1982.

5. W. Kaup, A Riemann mapping theorem for bounded symmetric domains in complex Banach spaces, Math. Z. 138 (1983), 503-529.

6. O. Loos, Bounded symmetric domains and Jordan pairs, Irvine Lecture Notes, 1977.

7. H. Upmeier, Symmetric Banach manifolds and Jordan $C^{*}$-algebras, North-Holland, Amsterdam, 1985.

8. H. Zettl, A characterization of ternary rings of operators, Adv. Math. 48 (1983), 117-143.

Department of Mathematics, University of California, Irvine, California 92717

E-mail address: brusso@math.uci.edu 